\title{
The Efficacy of Fampridine on Upper Extremity Functions in Individuals with Multiple Sclerosis: Is There a Difference between Cerebellar and Pyramidal Dysfunction?
}

\author{
(D) Asiye Tuba Ozdogar ${ }^{1 *}$, (D) Sinem Ozcelik2, (D) Seda Dastan 3 \\ 1Dokuz Eylul University Graduate School of Health Sciences, Izmir, Turkey \\ 2Dokuz Eylül University Faculty of Medicine, Department of Neurology, Izmir, Turkey \\ ${ }^{3}$ Dokuz Eylul University Graduate School of Health Sciences, Izmir, Turkey
}

\section{Abstract}

Objective: Similar to its positive effect on the lower extremities, fampridine can also affect upper extremity dysfunction. This study evaluated the potential effect of fampridine therapy on upper extremity functions in patients with multiple sclerosis (pwMS) by comparing these on the basis of cerebellar and pyramidal dysfunctions.

Materials and Methods: Patients aged between 18 and 60 years with a diagnosis of multiple sclerosis and eligible for fampridine therapy due to walking difficulties were followed-up. Of these, patients with problems such as lack of coordination in hand functions or deficiencies in fine motor skills, dressing, writing, and/or buttoning were invited to take part in the study. Upper extremity functions were evaluated using the 9-Hole Peg Test (9-HPT), and general disability was evaluated with the Expanded Disability Status Scale.

Results: One hundred fifty-nine patients were followed-up for 12 months, and 151 of them were included in the analysis. Seventy-seven (50 women) healthy controls ( $\mathrm{HCs}$ ) were also included. There was no statistically significant difference between the demographic characteristics of the two groups. A $19.8 \%$ improvement was observed in 9-HPT scores after one month of treatment ( $p=0.004)$. This improvement was observed to persist at the $24^{\text {th }}$ month. Patients with a cerebellar FS score of 0 to $2(n=76)$ improved significantly more $(p<0.001)$ than those $(23.5 \%)$ with a cerebellar FS score of 3 or higher (9.2\%).

Conclusion: The results of this study show that fampridine improves upper extremity functions in pwMS. This improvement was more pronounced in the group with cerebellar dysfunction.

Keywords: Multiple sclerosis, fampridine, upper extremity, disability level

\section{Introduction}

Upper extremity impairment is a common and disabling symptom reported in $66 \%$ of persons with multiple sclerosis (pwMS). The most extensive clinical signs and symptoms are loss of sensation, muscle weakness, tremor, loss of vibration sense, and reduced range of motion in the upper extremities. Bilateral upper limb disability occurs in more than half of pwMS (1). Manual dexterity is one of the most affected daily life activities in MS. Seventy-five percent of pwMS are reported to experience decreased manual dexterity, while 50\% experience limitations in daily life activities (2).
There is no cure for MS. Although the use of disease-modifying therapies reduces the rates of relapse and disability associated with the disease activity, symptomatic therapy is still a crucial component of treatment (3). The aim of several symptomatic treatments is therefore to reduce disease-related symptoms. However, there is still no proven symptomatic therapy capable of effectively treating upper limb impairment (4).

Fampridine is a voltage-dependent, specific blocker of neuronal fast potassium channels that promotes the transmission of neural flow through the demyelinated axons, thereby strengthening neuromuscular transmission (5). A previous

Address for Correspondence: Asiye Tuba Ozdogar, Dokuz Eylul University Graduate School of Health Sciences, Izmir, Turkey E-mail: asiye.tuba.ozdogar@gmail.com ORCID-ID: orcid.org/0000-0003-0043-9374

Received: 16.12.2021 Accepted: 27.01.2022

${ }^{\circ}$ Copyright 2021 by the Journal of Multiple Sclerosis Research published by Galenos Publishing House. 
review study has shown that fampridine exhibits substantial effects on the ability to walk short distances and on perceived walking capacity. However, other effects, such as improving visual function, spasticity, fatigue, quality of life, and upper limb and cognitive functions, remain unclear (6). Additionally, a recent meta-analysis has reported that fampridine can improve disability related to ambulation in MS (7).

Although fampridine appears to be effective in terms of gait and mobility, most studies did not investigate its effects against upper limb impairment, which commonly affects pwMS. Jensen et al. speculated that fampridine might be as effective in upper limb impairment as in the lower limbs (8). However, a recent systematic review investigating the effects of fampridine has reported no significant changes in terms of the Nine-Hole Peg Test (9-HPT) in either the short or long terms (6). Pickering et al. (9) has reported that fampridine does not affect ABILHAND scores. Marion et al. (4) has detected no significant improvement in 9-HPT grip strength, sensory function, or discrimination capacity. Upper extremity functions have been identified as a secondary outcome measure in the majority of studies. More case-control research is therefore needed for a better understanding of the effectiveness of fampridine on upper limb impairment in pwMS. The purpose of this study was to assess the effect of fampridine therapy on upper limb functions in pwMS.

\section{Materials and Methods}

\section{Participants}

This study was carried out in our multiple sclerosis (MS) clinic. The study protocol was approved by the Non-invasive Research Ethics Committee. Patients aged between 18 and 60 years with the diagnosis of MS (2017 McDonald criteria) and eligible for fampridine therapy due to walking difficulties were followedup. Those patients with problems such as lack of coordination in hand functions, or deficiencies in fine motor skills, dressing, writing and/or buttoning were invited to take part in the study. Patients who had experienced an attack in the last 30 days, who refused to provide written/verbal consent, or who were unable to complete the 12-month follow-up period for any reason were excluded from the study.

\section{Outcome Measures}

The participants' demographic and clinical data were collected. The Expanded Disability Status Scale (EDSS) is the most frequently used measure of disability in pwMS. Scoring is based on the neurological examination of seven functional systems, involving the patient's pyramidal, cerebellar, brainstem, sensory, bladder and bowel, visual, cerebral, and ambulatory status (10). The same senior neurologist examined the patients and calculated their global EDSS scores and cerebellar and pyramidal functional system scores. The 9-HPT is the most commonly used scale for evaluating upper extremity functions in pwMS
(11). The 9-HPT is valid and reliable in pwMS and is considered the gold standard for evaluating upper extremity functions (12). 9-HPT data were evaluated for the affected dominant hand.

\section{Procedure}

All participants were evaluated before fampridine therapy and after the first month of treatment. If the treatment was effective, they were also evaluated at the $3^{\text {rd }}, 6^{\text {th }}, 12^{\text {th }}$, and $24^{\text {th }}$ months in order to investigate the persistence of the effect. Age and sexmatched healthy controls were evaluated in the same periods in order to avoid the learning effect. Healthy relatives of patients admitted to the hospital and who agreed to participate in the study were included as the healthy control group.

\section{Statistical Analysis}

Data were analyzed on IBM SPSS 2019 Statistics for Windows software. The Shapiro-Wilk test and histogram graphs were employed to evaluate whether the data were normally distributed. Descriptive analyses were presented using mean plus standard deviation for continuous variables and numbers and percentages for categorical variables. Parametric tests were applied since all data were normally distributed.

\section{Results}

One hundred fifty-nine patients were followed-up for 12 months, 151 of them were followed-up for 24 months after the initiation of treatment and were included in this study. Seventy-seven (50 female) healthy controls were also enrolled. No significant statistical differences were observed in terms of demographic characteristics between these two groups (Table 1). The pre-treatment mean EDSS score of the study group was 5.3 , which decreased to 5.1 in the first month ( $p=0.002$ ). The mean EDSS scores also remained stable for two years (Figure 1). The mean pyramidal functional scores were $3.22 \pm 1.7$ in the group with cerebellar functional scores of $0-2$ and $3.52 \pm 2.1$

\begin{tabular}{|c|c|c|c|}
\hline & $\begin{array}{l}\text { Patients } \\
\text { with MS } \\
(n=159)\end{array}$ & $\begin{array}{l}\text { Healthy } \\
\text { control } \\
(n=77)\end{array}$ & $p$ value \\
\hline Female, n (\%) & $111(69.8 \%)$ & 50 (64.9\%) & NS \\
\hline Age (years) & $45.5 \pm 8.1$ & $46.4 \pm 7.9$ & NS \\
\hline Education (years) & $11.3 \pm 3.8$ & $11.7 \pm 4.1$ & NS \\
\hline $\begin{array}{l}\text { EDSS (mean) } \\
\text { Baseline } \\
24^{\text {th }} \text { month }\end{array}$ & $\begin{array}{l}5.3 \\
5.1 \\
\mathbf{0 . 0 0 2}\end{array}$ & - & \\
\hline $\begin{array}{l}\text { 9-HPT score (mean) } \\
\text { Baseline } \\
24^{\text {th }} \text { month }\end{array}$ & $\begin{array}{l}19.8 \\
17.6 \\
\mathbf{0 . 0 0 4}\end{array}$ & $\begin{array}{l}14.2 \\
14.08 \\
>0.05\end{array}$ & \\
\hline
\end{tabular}

EDSS: Expanded Disease Status Scale, 9-HPT: Nine-Hole Peg test, MS: Multiple sclerosis, NS: Not significant

Significant values are shown in bold and italic. 
in the group with a cerebellar functional scores of 3 or above $(p=0.08)(p>0.05)$. However, the patient group with cerebellar functional system scores of $0-2$ exhibited significantly greater improvement than the patients (23.5\%) with cerebellar functional system scores of 3 or more $(9.2 \%)(p<0.001)$.

9-HPT scores, which are frequently used to evaluate the effects of both pyramidal and cerebellar functions on the upper extremity, were evaluated regularly before treatment and during follow-up. 9-HPT scores improved from 19.8 to 17.3 after one month of treatment $(\mathrm{p}=0.004)$. This improvement was also sustained in months 3, 6, 12, and 24 (Figure 2). The healthy controls' 9-HPT results also improved (3.6\%), although this was not statistically significant ( $p>0.05)$.

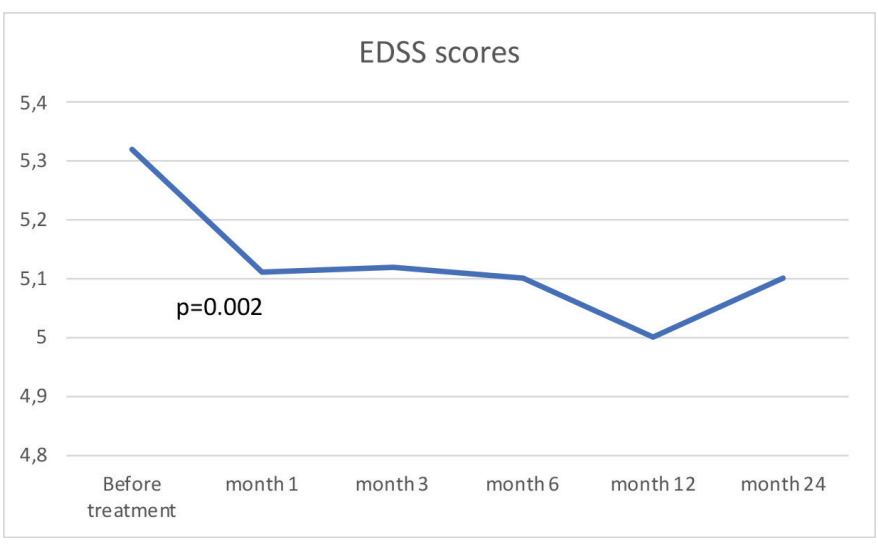

Figure 1. EDSS scores at follow-up in fampridine group

EDSS: Expanded Disease Status Scale

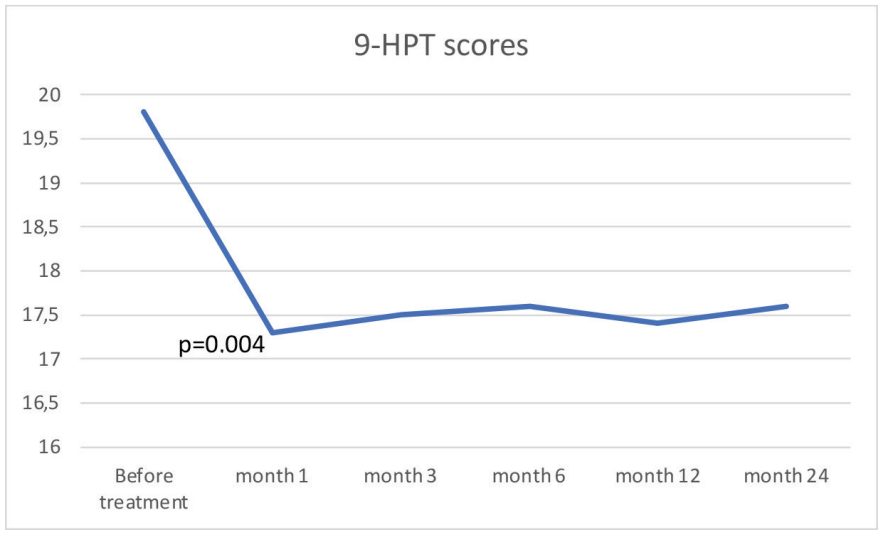

Figure 2. 9-HPT scores at follow-up in fampridine group 9-HPT: Nine-Hole Peg Test

\section{Discussion}

Walking disorders due to causes such as weakness in the lower extremities, spasticity, cerebellar disorder, or profound sensory impairment are prominent symptoms in the great majority of individuals with MS. However, impairments in upper extremity functions secondary to tremor, lack of coordination, and paresis can also be observed in the course of the disease in most
pwMS. Similar to lower extremity functions, upper extremity functions also restrict patients'activities of daily living. Although this occurs more in the progressive phase, hand functions may still be affected at a high rate in the early period. The therapeutic options for coping with the deterioration of fine motor skills, which is often not noticed by patients and their relatives, are limited.

Two of the well-defined disadvantages of the EDSS are its inability to assess upper extremity functions and its low reproducibility. The EDSS does not include upper extremity dysfunction after certain levels of disability have been reached but is used as an ambulation index. Differences also occur among practitioners. Although a number scales are used to evaluate upper extremity functions, the most commonly used tool in MS is 9-HPT. Studies have mainly concentrated on the effects of fampridine on the walking abilities of pwMS, and only limited studies have focused on its effects on upper extremity functions as a primary endpoint. The data concerning the effects of fampridine on upper extremity functions in these studies are also inconsistent (6).

The main finding of this study is that fampridine provides effective symptomatic treatment in the context of upper extremity functions in pwMS compared to healthy controls. Moreover, the effect of the treatment persists for up to 24 months. Fampridine does not exhibit selective actions on nerve fibers, and it may be expected to work on all fibers and to increase nerve transmission. A recent systematic review investigating the effects of fampridine in pwMS has included four studies that assessed upper extremity functions. All four used the 9-HPT to evaluate upper extremity functions, and all reported no significant changes $(8,9,13,14)$. In contrast, similarly to our own results, Savin et al. (15) reported improvement in 9-HPT after three months of fampridine therapy. Such inconsistent results may be attributed to the differences in patient selection and disability levels.

MS relapse can occur in any part of the central nervous system, with no preference being exhibited. Long tracts may therefore be expected to have a greater lesion load than comparatively shorter fibers, due to their greater surface area and higher probability of demyelination. The lower extremities of pwMS may therefore be affected more than the upper extremities. For the most part, problems with upper extremity functions become noticeable in the advanced stages of the disease when the patient is already restricted to a wheelchair. This may explain the lack of significant improvement in upper extremity functions after fampridine trials in patients with lower disability levels. It would therefore be logical to select patients with decreased upper extremity functions, which can be determined by performing baseline measurements. In other words, we would expect the patient to improve with fampridine therapy if a loss of function is already present. The healthy controls in 
the present study exhibited no significant improvement in their 9-HPT results. A healthy control group was also established to assist with controlling the learning factor. Patients with lower disability levels on the cerebellar EDSS subscore improved more than those with higher disability levels. Cerebellar dysfunction can alter motor functions through loss of coordination. We hypothesize that improved motor functions may overcome lower disability levels on cerebellar functional scores. Furthermore, improved motor performance was unable to compensate for higher cerebellar disability.

\section{Study Limitations}

The strength of this study is that it presents patients' long-term results between the period before the start of drug therapy and up to the $24^{\text {th }}$ month of use. The study findings showed that although the maximum improvement was seen in the first month, the effect of treatment also persisted in the $24^{\text {th }}$ month. No improvement was observed in 9-HPT, such as indicating a learning effect in the healthy control group. The main limitation of this study was the absence of a control group of pwMS who had never used fampridine for the purpose of comparing upper extremity functions. The lack of analysis by classifying cases as progressive or non-progressive may also be a limiting factor. A final limitation is that data for disease modifying drugs (DMDs) used by patients were not included in the analysis.

\section{Conclusion}

Fampridine caused an improvement in the upper extremity functions of PwMS, and its positive effect lasted for up to 24 months. Patients with more severe cerebellar dysfunction exhibited less improvement in upper extremity functions than the group with mild cerebellar dysfunction.

Acknowledgments: The authors acknowledge the Multiple Sclerosis Research Association for assistance during the recruitment of the study.

\section{Ethics}

Ethics Committee Appproval: Ethics committee approval for this study was obtained from the Local Ethics Committee of Dokuz Eylul University. The study protocol was approved by the Non-invasive Research Ethics Board (protocol number: 3696GOA, approval Number: 2017/29-10).

Informed Consent: Written consent received from all participants.

Peer-review: Externally and internally peer-reviewed.

\section{Authorship Contributions:}

Concept: B.P.C., S.O., Design: B.P.C., S.O., Data Collection or Processing: A.T.O., T.K., Analysis or Interpretation: A.T.O., T.K., S.O., Literature Search: A.T.O., T.K., Writing: B.P.C., A.T.O., T.K., S.O. S.D.
Conflict of Interest: The authors declare that they have no known competing financial interests or personal relationships that could have appeared to influence the work reported in this paper.

Financial Disclosure: This research did not receive any specific grant from funding agencies in the public, commercial, or notfor-profit sectors.

\section{References}

1. Bertoni R, Lamers I, Chen CC, Feys P, Cattaneo D. Unilateral and bilateral upper limb dysfunction at body functions, activity and participation levels in people with multiple sclerosis. Multiple Scler 2015;21:1566-1574.

2. Johansson S, Ytterberg C, Claesson IM, Lindberg J, Hillert J, Andersson M, Widén Holmqvist L, von Koch L. High concurrent presence of disability in multiple sclerosis. Associations with perceived health. J Neurol 2007;254:767-773.

3. Wingerchuk DM, Weinshenker BG. Disease modifying therapies for relapsing multiple sclerosis. BMJ 2016;354:3518

4. Marion S, Leonid C, Belinda B, Joanne D, Elise H, Leeanne C, et al. Effects of modified-release fampridine on upper limb impairment in patients with Multiple Sclerosis. Mult Scler Relat Disord 2020;40:101971.

5. Ruck T, Bittner S, Simon OJ, Göbel K, Wiendl H, Schilling M, Meuth SG. Longterm effects of dalfampridine in patients with multiple sclerosis. J Neurol Sci 2013;337:18-24.

6. Valet M, Quoilin M, Lejeune $T$, Stoquart G, Van Pesch V, El Sankari S Detrembleur C, Warlop T. Effects of Fampridine in People with Multiple Sclerosis: A Systematic Review and Meta-analysis. CNS Drugs 2019;33:10871099.

7. Shi J, Wu X, Chen Y. Study on Dalfampridine in the treatment of Multiple Sclerosis Mobility Disability: A meta-analysis. PLos One 2019;14:e0222288.

8. Jensen HB, Nielsen JL, Ravnborg M, Dalgas U, Aagaard P, Stenager E. Effect of slow release-Fampridine on muscle strength, rate of force development, functional capacity and cognitive function in an enriched population of MS patients. A randomized, double blind, placebo controlled study. Mult Scler Relat Disord 2016;10:137-144.

9. Pickering H, Murray J, Lin CS, Cormack C, Martin A, Kiernan MC, Krishnan AV. Fampridine treatment and walking distance in multiple sclerosis: A randomised controlled trial. Clin Neurophysiol 2017;128:93-99.

10. Kurtzke JF. Rating neurologic impairment in multiple sclerosis: an expanded disability status scale (EDSS). Neurology 1983;33:1444-1452.

11. Cutter GR, Baier ML, Rudick RA, Cookfair DL, Fischer JS, Petkau J, Syndulko K, Weinshenker BG, Antel JP, Confavreux C, Ellison GW, Lublin F, Miller AE, Rao SM, Reingold S, Thompson A, Willoughby E. Development of a multiple sclerosis functional composite as a clinical trial outcome measure. Brain 1999;122:871-882

12. Lamers I, Kelchtermans S, Baert I, Feys P. Upper limb assessment in multiple sclerosis: a systematic review of outcome measures and their psychometric properties. Arch Phys Med Rehabil 2014;951184-1200.

13. Goodman AD, Brown TR, Cohen JA, Krupp LB, Schapiro R, Schwid SR, Cohen R, Marinucci LN, Blight AR; Fampridine MS-F202 Study Group. Dose comparison trial of sustained-release fampridine in multiple sclerosis. Neurology 2008;71:1134-1141.

14. Goodman AD, Cohen JA, Cross A, Vollmer T, Rizzo M, Cohen R, Marinucci L, Blight AR. Fampridine-SR in multiple sclerosis: a randomized, double-blind, placebo-controlled, dose-ranging study. Mult Scler 2007;13:357-368.

15. Savin Z, Lejbkowicz I, Glass-Marmor L, Lavi I, Rosenblum S, Miller A. Effect of Fampridine-PR (prolonged released 4-aminopyridine) on the manual functions of patients with Multiple Sclerosis. J Neurol Sci 2016;360:102-109. 